\title{
Optimization of Supply Diversity for the Self-Assembly of Simple Objects in Two and Three Dimensions
}

\author{
Fabio R. J. Vieira \\ Valmir C. Barbosa* \\ Universidade Federal do Rio de Janeiro \\ Programa de Engenharia de Sistemas e Computação, COPPE \\ Caixa Postal 68511 \\ 21941-972 Rio de Janeiro - RJ, Brazil
}

\begin{abstract}
The field of algorithmic self-assembly is concerned with the design and analysis of self-assembly systems from a computational perspective, that is, from the perspective of mathematical problems whose study may give insight into the natural processes through which elementary objects self-assemble into more complex ones. One of the main problems of algorithmic self-assembly is the minimum tile set problem (MTSP), which asks for a collection of types of elementary objects (called tiles) to be found for the self-assembly of an object having a pre-established shape. Such a collection is to be as concise as possible, thus minimizing supply diversity, while satisfying a set of stringent constraints having to do with the termination and other properties of the self-assembly process from its tile types. We present a study of what we think is the first practical approach to MTSP. Our study starts with the introduction of an evolutionary heuristic to tackle MTSP and includes results from extensive experimentation with the heuristic on the self-assembly of simple objects in two and three dimensions. The heuristic we introduce combines classic elements from the field of evolutionary computation with a problem-specific variant of Pareto dominance into a multi-objective approach to MTSP.
\end{abstract}

Keywords: Algorithmic self-assembly, Minimum tile set problem, Multiobjective evolutionary algorithms.

\footnotetext{
*Corresponding author (valmir@cos.ufrj.br).
} 


\section{Introduction}

Self-assembly is the process whereby simple building blocks self-organize into more complex structures. Its occurrence in nature is ubiquitous and can be observed at various scales, ranging from the growth of crystals, through the production of complex molecules inside the living cell, to the formation of galaxies and even their clustering into larger structures. In recent years, a considerable amount of research has been directed toward attempting to understand and reproduce the essential mechanisms that drive self-assembly, aiming at amassing its power of distributed control for goal-directed building tasks of strategic importance [12, 9. The premise has been that, if successful, such efforts may eventually lead to autonomous systems that, for example, will replace the current process of photolithography in VLSI fabrication [11, or the current electronic media for information storage [15], or yet will give rise to cooperating teams of robots for nano- and large-scale construction through rules of self-assembly 20, 22] or to solvers of hard mathematical problems through DNA computing 23, 4, 5. At the current stage, a lot of research is being directed toward the biochemical production of nanoscale building blocks [26, 27, 15, 14, 10] or devices [11, 15, 17, or the elaboration of logical rules for the programming of robots [18, 22.

The mathematical study of self-assembly can be said to have begun as far back as 21] on the formation of carbon crystals from the nanoscale structures that the author called tiles. However, it seems to have been only much more recently, after algorithm- and complexity-related notions were sufficiently mature from their development within computer science, that momentum began to accumulate. The resulting discipline is currently known as algorithmic selfassembly. It is based on the two-dimensional model laid down in [24, 16], itself an extension of the earlier, one-dimensional model of [1]. The model is built on an unbounded two-dimensional grid at whose nodes square tiles can be placed so that tiles that occupy neighboring nodes are themselves juxtaposed with a side in common. Each tile is labeled on at least one of its four sides and to each label there corresponds a unique positive integer indicating the intensity that the bond formed by juxtaposing two tiles on sides of equal labels will have. Tiles may occur in a finite number of types, each capable of supplying an unbounded number of identical tiles.

In this model, the process of self-assembly begins with the placement of a special tile, called the seed, at an arbitrary node. It then proceeds in discrete time steps by accreting one tile of one of the available types per time step. It is important to note that growth can only take place by accretion, that is, by aggregation to the exterior of the current object, which expressly prevents hollows that are left from being filled later on. The model also includes a positive integer parameter, called a temperature, intended to regulate the addition of new tiles. Specifically, a new tile may only be added if bonds are formed only on sides of the same label, and furthermore the net intensity of the resulting (at

most three) bonds surpasses the temperature. A sequence of two-dimensional objects is then established of which the first comprises the seed tile only and 
each of the others is the augmentation of its precursor by the accretion of exactly one tile.

A considerable body of knowledge has been developed on this model, targeting primarily the establishment of theoretical properties of the objects produced from both a structural and a functional perspective [6, 2, 13, 3, 25. Along with these properties, optimization problems have been defined that embody the essential difficulties associated with translating all the theoretical discoveries into the practical context that motivated the whole field in the first place. One of these problems is that of finding out how diverse the supply of tiles for self-assembly has to be in order for an object of pre-established shape to be produced. In other words, the problem asks for the minimum number of tile types for assembling an object of the desired shape.

This problem is known as the minimum tile set problem (henceforth, MTSP) [1. Given a temperature and a two-dimensional shape, MTSP asks for a seed tile and the least diverse set of tile types out of which an object of the desired shape may be assembled. MTSP is constrained by the requirements that all object sequences obtained from the seed terminate, that they all terminate in an object of the desired shape, and furthermore that the objects obtained at termination have the property of being full (in the sense of containing as many bonds as there can be juxtaposed square sides). Formally, MTSP is an NP-hard problem [1. While to the practitioner this is generally an indication of inherent computational difficulty and a clear sign that heuristics are to be sought to solve the problem, in the case of MTSP even choosing an adequate heuristic has the feel of something arduous, owing in essence to the fact that the problem's constraints (termination, unicity, and fullness) seem to loom too large upon the computational resources one can usually count on.

Our subject in this paper is the development of a heuristic to tackle MTSP. The heuristic we describe is an evolutionary algorithm for multi-objective optimization: it uses a convenient variation of Pareto dominance along with suitable crossover and mutation operators to drive the search in the direction of concise tile sets that do nonetheless satisfy the problem's constraints. We report on experiments for the two-dimensional model discussed above, for a variation thereof that allows tiles to be rotated on the plane before being accreted onto the growing object, and likewise for the three-dimensional extension of the twodimensional model with rotation. These two additional models appear, to our knowledge, nowhere else in the literature on algorithmic self-assembly. They seem, however, to enable more realistic representations of real-world processes of self-assembly, as argued in [8] in the case of three dimensions, and have for this reason been included.

We are aware of no other study targeting a practical approach to MTSP1 While this is surprising per se, in the context of the present paper it has also hindered the possibilities for a comparative assessment of our heuristic vis-à-

\footnotetext{
${ }^{1}$ The work reported in 19 is the only one to come somewhat close to being an exception, but its relation to our work remains very tenuous, since the problem that is handled there does not share the objective or constraints of MTSP, even though it too targets the determination of tile types.
} 
vis others severely. On the computational side, then, we have concentrated on squares and cubes as the desired final shapes. For the original two-dimensional model, at least, this has given us a solid basis for comparison, since upper bounds on the number of necessary tile types are available from [16. For the other two models no such knowledge is available, but we expect relatively smaller tile sets to suffice when the possibility of rotation is added to the two-dimensional model, which then gives us an indirect basis on which to judge what we find in this case. As for the three-dimensional case, all we can achieve at this point is a qualitative evaluation with respect to the two-dimensional cases.

We proceed in the following manner. We first detail MTSP and also the three models we use in Section 2. Then we present our heuristic with all its elements in Section 3 and computational results in Section 4. Further comments and concluding remarks are given in Section 5 .

\section{The Minimum Tile Set Problem}

\subsection{Problem Formulation}

The formulation of MTSP we give in this section is good for the two-dimensional models and for the three-dimensional model. We use the word tile to refer both to a square and to a cube, and likewise the word side to refer both to a side of a square and a face of a cube. We do this for conciseness and no confusion should arise.

Let $\mathcal{T}$ denote a set of tile types and let $S$ be the seed tile. The process of self-assembly from $S$ and $\mathcal{T}$ assumes the existence of an unbounded supply of tiles of all types in $\mathcal{T}$ and is represented by a sequence $O=\left\langle\omega_{1}, \omega_{2}, \ldots\right\rangle$, where $\omega_{1}$ is the object constituted by the single tile $S$. For $u>1, \omega_{u}$ is the object obtained from $\omega_{u-1}$ by the accretion of exactly one tile of one of the types in $\mathcal{T}$ for which model-dependent binding conditions are satisfied. Such conditions will be specified shortly for each model, but they all have in common the property that, as the new tile is accreted onto $\omega_{u-1}$, a number of bonds is created that is at least one and at most the number of sides in the tile through which binding can occur (provided at least one of the tile's sides is left unbound, given that growth is effected by accretion). If $t$ is a tile in the resulting $\omega_{u}$, we let $b_{u}(t)$ denote the number of bonds involving $t$ in $\omega_{u}$. Similarly, we let $B_{u}$ be the total number of bonds holding $\omega_{u}$ together. Clearly, $B_{u}=\sum_{t} b_{u}(t) / 2$, where the summation ranges over all tiles in $\omega_{u}$.

Now let $\Omega_{S}$ be the set of all sequences that represent a self-assembly process from $S$. Also, let $\omega^{*}$ be any object having the desired, pre-specified shape and let $B^{*}$ be the maximum number of bonds that may exist in an object of that shape. If $\tau>0$ is the temperature, then MTSP requires that $S$ and $\mathcal{T}$ be determined such that $|\mathcal{T}|$ is minimum over all seeds and tile-type sets that satisfy the following constraints:

C1. (Termination) For all $O \in \Omega_{S}, O$ is finite; let $|O|$ be the number of objects in $O$. 
C2. (Unicity) For all $O \in \Omega_{S}, \omega_{|O|}$ has the same shape as $\omega^{*}$.

C3. (Fullness) For all $O \in \Omega_{S}, B_{|O|}=B^{*}$.

Notice, in this formulation, that the temperature $\tau$ is never mentioned explicitly. This is because the role it plays is in helping specify the binding conditions that allow a new tile to be accreted onto an object. These conditions are given below as part of each model's characteristics, but the way they relate to $\tau$ is common to all three models. We handle this before discussing the models.

Each tile type $T \in \mathcal{T}$ is characterized by a number $\sigma(T)$ of sides (either 4 or 6 , respectively in two and three dimensions) and by a number $\lambda(T)$ of sides through which binding is possible. These numbers are such that $1 \leq \lambda(T) \leq \sigma(T)$. Each of the $\lambda(T)$ sides is labeled to indicate how binding through that side does actually take place. If $\ell$ is one such label, then whenever binding occurs through a side that is thus labeled the resulting bond has intensity given by the integer $I(\ell)>0$. The $\tau$-dependent condition for a tile $t$ of type $T$ to be accreted onto an object at a certain place is that $I\left(\ell_{1}\right)+\cdots+I\left(\ell_{\lambda(t)}\right) \geq \tau$, where $\ell_{1}, \ldots, \ell_{\lambda(t)}$ are the labels on the sides through which binding does take place (and therefore $\lambda(t) \leq \lambda(T))$. What is left to specify of each model's binding conditions is precisely the $\lambda(t)$ sides.

\subsection{Two-Dimensional Model (2D)}

This is the original model of [16 and is characterized by a fixed orientation of all tile types with respect to the underlying two-dimensional lattice. That is, if we identify "north," "east," "south," and "west" directions on the lattice and proceed likewise on all tile types, then a tile may only be accreted onto a growing object if its directions are aligned with those of the lattice. This given, the binding conditions for this model require identical labels on the sides that face each other for a bond to be created between them. Thus, accreting a tile $t$ onto an object requires $\lambda(t) \leq 3$ pairs of matched labels. This is illustrated in Figure $1{ }^{2}$

The 2D model may seem too restrictive or even implausible, but from [16 we know what seems to be tight upper bounds on the value of the optimal $|\mathcal{T}|$ when the desired shape is that of an $n \times n$ square with $n \geq 3$. The $2 \mathrm{D}$ model is then invaluable in our present context, as it provides the only available benchmark with which the results we obtain can be compared. What is demonstrated in [16] is that, for $\tau=1$, and letting $\mathcal{T}^{*}$ be an optimal tile set, $\left|\mathcal{T}^{*}\right| \leq n^{2}$. For $\tau=2$,

$$
\left|\mathcal{T}^{*}\right| \leq \begin{cases}n+4, & \text { if } 3 \leq n \leq 23 \\ 22+\left\lceil\log _{2} n\right\rceil, & \text { if } 23 \leq n<22+2^{23} \\ 22 \log _{2}^{*} n, & \text { if } 22+2^{23} \leq n\end{cases}
$$

\footnotetext{
${ }^{2}$ In this figure, and in others to follow for the two-dimensional cases, juxtaposed tiles are represented with a gap between them so that the matching labels can be shown explicitly whenever possible.
} 


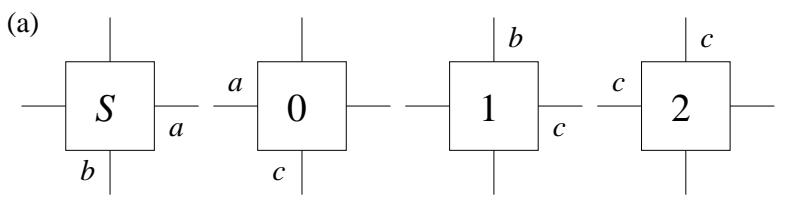

(b)

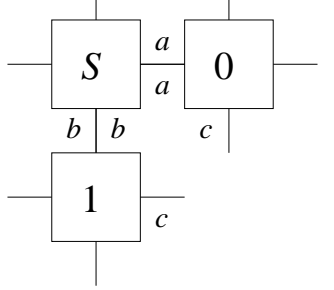

(c)

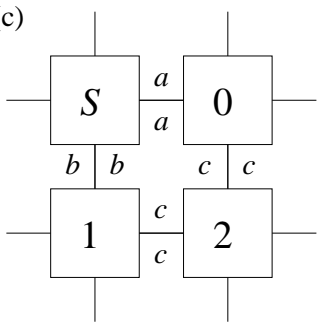

Figure 1: In the 2D model, using the tile types in (a) allows the object in (c) to be obtained from the object in (b) by accreting a tile of type 2 onto it. Temperature is $\tau=2$. Label intensities are $I(a)=I(b)=2$ and $I(c)=1$.

( $\log _{2}^{*}$ indicates the number of times one must apply the $\log _{2}$ operator in succession until the result is no greater than 1). As for $\tau \geq 3$, it is unknown whether the bounds in (11) can be improved.

\subsection{Two-Dimensional Model with Rotation (2DR)}

Our main motivation to introduce the 2DR model has been the possibility of increased plausibility that comes with allowing clockwise or counterclockwise rotation of a tile by multiples of $90^{\circ}$ before accreting it onto an object 3 Rotations are intrinsically problematic, though: as shown in Figure 2 there exist tile-type sets that, by virtue of the added possibility of rotation, make it impossible for constraint $\mathrm{C} 1$ to be satisfied. We circumvent this problem by adopting the binding conditions of the $2 \mathrm{D}$ model and making them more stringent. The idea is to let each label have a polarity (either + or - ) and to require different polarities, in addition to equal labels, for the bond to be created. Similarly to the $2 \mathrm{D}$ model, accreting a tile $t$ onto an object requires $\lambda(t) \leq 3$ pairs of matched labels, each pair with different polarities. This is also illustrated in Figure 2,

Unlike the 2D model, there are now no known upper bounds on $\left|\mathcal{T}^{*}\right|$. But the 2DR model is more flexible than the 2D model, in the sense that, in principle, the same tile type may be used in different situations, depending on how tiles are rotated. In fact, each tile type in 2DR stands for up to 4 distinct tile types in $2 \mathrm{D}$, so we expect solutions to be no worse than in the $2 \mathrm{D}$ case, possibly even better.

\footnotetext{
${ }^{3}$ Notice that this is the only kind of rotation that makes sense in two dimensions. Possibilities like flipping a tile around its north-south or east-west axis, for example, implicitly require incursions into the third dimension and must not be considered.
} 
(a)

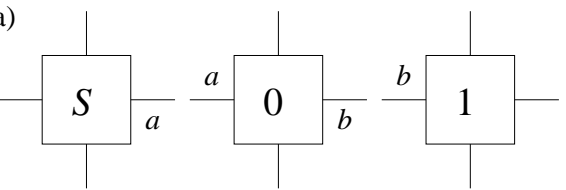

(c)

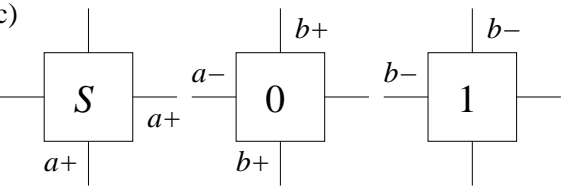

(d)

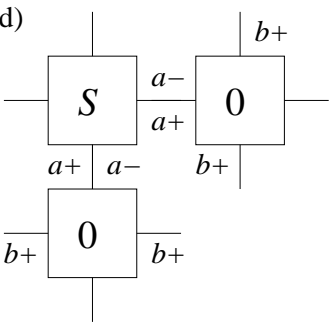

(b)

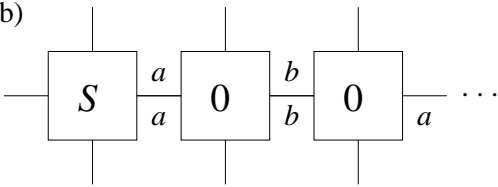

(e)

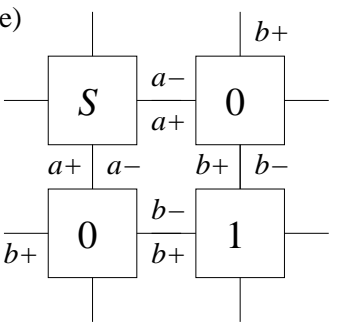

Figure 2: In the 2DR model, using the tile types in (a) may lead to the unbounded growth shown in (b). Using the tile types in (c), where polarities are also used, allows the object in (e) to be obtained from the object in (d) by accreting a tile of type 1 onto it. Temperature is $\tau=2$. Label intensities are $I(a)=I(b)=2$ in (a) and (b), $I(a)=2$ and $I(b)=1$ in (c)-(e). 


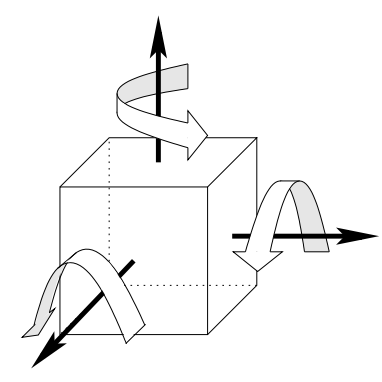

Figure 3: A tile's possible rotations in the 3DR model.

\subsection{Three-Dimensional Model with Rotation (3DR)}

As we move into three dimensions, motivation is derived directly from the field of molecular dynamics [7 and, in the absence of any upper bounds like those given in (11) for the $2 \mathrm{D}$ model, it is very hard to justify the impossibility of rotation. We have then skipped what would be a 3D model and moved directly to 3DR, three-dimensional with the possibility of rotation. The underlying lattice is now three-dimensional and the possible rotations are by multiples of $90^{\circ}$ around one of the three possible axes, as shown in Figure 3 . As a consequence, each tile type in 3DR stands for up to 24 distinct tile types in the (hypothetical) 3D model.

Rotations are just as problematic in 3DR as they are in $2 \mathrm{DR}$, and for the same reason. We then continue to use label polarity, so that accreting a tile $t$ onto an object now requires $\lambda(t) \leq 5$ matching label pairs, each with different polarities, as illustrated in Figure 4. The 3DR model also shares with the 2DR model the absence of benchmarks against which to experiment, but as we move from square shapes in two dimensions to cubic shapes in three dimensions an interesting trade-off turns up. On the one hand, the added third dimension allows for more complex shapes to be considered; on the other hand, rotating tiles have more degrees of freedom in three dimensions than in two. Just which trend may eventually have the upper hand is unclear now, but we are given some basis for evaluation in three dimensions, at least in qualitative terms.

\section{An Evolutionary Heuristic}

\subsection{Overview}

Our heuristic is evolutionary and follows one of the customary layouts of a generational genetic algorithm running on a population of tile-type sets, each possibly of a different size. It runs for a fixed number of generations, each corresponding to a population of fixed size, the first population created randomly. Each of the subsequent populations is obtained from its precursor by first an elitist step that seeks to preserve some of the fittest individuals, then a diversity- 
(a)
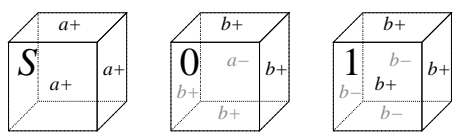

(b)

(c)
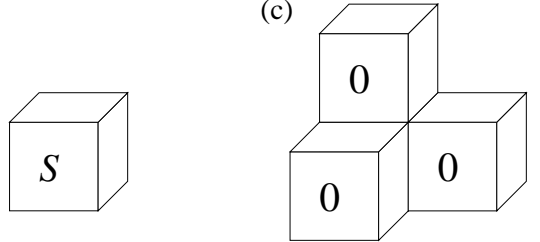

(d)

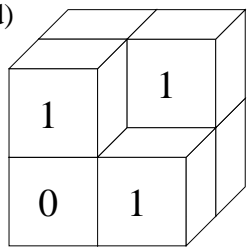

(e)

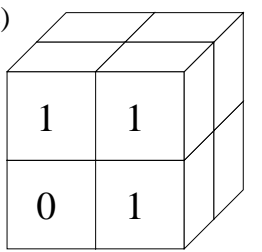

Figure 4: In the 3DR model, using the tile types in (a) allows the object in (e) to be obtained from the object in (b) through the following sequence of accretions: first three tiles of type 0 are accreted, one at a time, onto the growing object that results in the one in (c); then three tiles of type 1 are used to lead to the object in $(\mathrm{d})$; then a fourth object of type 1 is used. Temperature is $\tau=2$. Label intensities are $I(a)=2$ and $I(b)=1$. 
preserving step that samples the previous population uniformly at random and moves the resulting individuals to the new one, and then a step that makes use of crossover and mutation and is repeated until the new population is full. The diversity-preserving step never chooses from those individuals already picked by the elitist step.

At each repetition of the crossover- and mutation-based step, a choice is made as to whether the crossover or the mutation operator will be applied. In the former case, two individuals are selected and combined, and the resulting two individuals are added to the new population. In the latter case, one single individual is selected and mutated before being added to the new population. The choice is probabilistic and we have found that increasing the probability of choosing crossover from generation to generation sometimes provides the best results. If $G$ is the number of generations and $p_{g}$ is the probability of choosing crossover in the $g$ th generation, $1 \leq g \leq G$, then we use

$$
p_{g}=p_{1}+(g-1)\left(\frac{p_{G}-p_{1}}{G-1}\right),
$$

that is, we let the crossover probability increase linearly from the initial value $p_{1}$ through the final value $p_{G}$.

We do fitness-based selection, both in the elitist step and in each of the steps that choose between crossover and mutation, based on a multi-objective approach. While selecting from the population of generation $g$, we first group the individuals into layers of Pareto-like dominance, then we choose one of the layers probabilistically in a linearly normalized fashion, and then we select an individual from the chosen layer uniformly at random. If $L_{g}$ denotes the number of layers at this generation and $W_{g}$ the factor by which the top layer (the most dominant, assumed to be layer 1) is more likely than the bottom layer (the least dominant, assumed to be layer $L_{g}$ ) to be chosen, then the probability that the $l$ th layer is chosen, $1 \leq l \leq L_{g}$, is proportional to $W_{g}-(l-1)\left(W_{g}-1\right) /\left(L_{g}-1\right)$. We have also found that the best results often come from letting $W_{g}$ increase from generation to generation, since in the earliest generations the fitness components vary little from one individual to another and the layering process is practically devoid of meaning. As in the case of the crossover probability, we let it increase linearly from the initial value $W_{1}$ through the final value $W_{G}$, that is,

$$
W_{g}=W_{1}+(g-1)\left(\frac{W_{G}-W_{1}}{G-1}\right) \text {. }
$$

\subsection{The Individual and its Simulation}

Each individual represents a set of tile types whose size lies somewhere between a minimum and a maximum value, these bounds being the same for all individuals. Each tile type is characterized by a set of labels and, for each label, an intensity. During the evolutionary process there are two occasions in which labels must be selected randomly from some universe of possibilities: the first of these is the formation of the initial population; the second is when individuals 
are to be mutated. For use in these occasions, a fixed table of labels and associated intensities is used. Given the temperature $\tau$, this table includes a certain variety of labels for each of the possible intensities $1, \ldots, \tau$. It also includes the special label $\epsilon$, used to denote the absence of a label. Just how much variety is included depends on the size of the table, which is fixed beforehand. Given this size, the actual table is obtained by truncating the sequence of distinct labels $\epsilon, \ell_{1}, \ldots, \ell_{\tau}, \ell_{\tau+1}, \ldots, \ell_{2 \tau}, \ldots$ to as many labels as that desired size. As for the intensities of the non- $\epsilon$ labels, we let $I\left(\ell_{k \tau+1}\right)=1, \ldots, I\left(\ell_{(k+1) \tau}\right)=\tau$ for $k=0,1, \ldots$. Labels in this table have no polarities: if in the 2DR or 3DR model, polarities are chosen at random after each label has itself been chosen.

In order for an individual to be evaluated, we need to find out how close it comes to allowing an object of the desired shape to be assembled, and also how close it comes to satisfying the remainder of constraints C1 through C3. The first step toward these goals is to simulate the process of self-assembly for the individual in question, sometimes more than once, since it is unavoidable that each simulation be essentially of a stochastic nature. The results of the simulations can then be fed into appropriate fitness components, which in turn can be used to perform the desired evaluation.

Each simulation is carried out on a lattice with periodic boundaries having the same size along each dimension 4 The use of such boundaries allows the seed $S$ to be placed arbitrarily on the lattice, which is necessarily finite for the purposes of simulation. With $S$ in place, the simulation proceeds by maintaining two lists and managing the interactions between them. At all times, the first list contains all the lattice positions at which it is currently possible to add a new tile to the growing object without making it collide with itself along one of the dimensions. This list includes positions inside hollows, but this is not detrimental to the notion of growth by accretion, as we discuss shortly. The second list, in turn, contains all tiles that can currently be added to at least one of the available lattice positions. Each tile type of the individual under consideration may be represented in this list by several tiles, one for each of the possible lattice positions at which it can be placed and for each possible rotation (if $2 \mathrm{DR}$ or $3 \mathrm{DR}$ is the model in use). All that is required of each such tile is that it satisfy the binding conditions for the model under which the simulation is being conducted and also that all pertinent intensities add up to at least $\tau$. Also, if the lattice position to which the tile corresponds is inside a hollow, all these requirements must not involve a necessary bond with the tile that was the one to close the hollow.

The simulation repeatedly selects a matching pair of elements from the two lists, places the corresponding tile at the corresponding lattice position, and updates the lists. The selection is probabilistic and is done proportionally to the added intensities of the bonds to be created by each pair. This is done until the tile list becomes empty or a pre-specified maximum number of tiles has been used. If exit occurs on the former condition and the possibility of a collision

\footnotetext{
${ }^{4}$ Periodic boundaries are such that traversing the lattice along any dimension necessarily leads to an already visited node.
} 
never occurred, then the simulation is said to have reached a terminal object (this is not to say that the simulation would not terminate if it were allowed to continue past the maximum number of tiles, or if the lattice were infinite and collisions impossible, but merely reflects an arbitrary decision aimed at keeping the simulation bounded in both time and space).

It is important to note that, when a tile is placed inside a hollow during the simulation, this by no means indicates that the requirement of growth by accretion only has been given up. Because the bond, if any, created between such a tile and the one that closed the hollow earlier in the simulation is inessential (i.e., the tile could be placed in the hollow and satisfy all requirements even without such a bond), it is possible to accrete both tiles onto the object at the same positions but in reversed order and still obtain the same final result. The possibility of placing tiles inside hollows during simulation is then only a device to help reorder accretive additions to the growing object when they happen to be selected in an unfavorable order.

The simulation is repeated until a terminal object is reached or else a prespecified number of simulations has been performed. In the former case, the results to be fed into the fitness components are obtained from the terminal object that was reached. In the latter case, they are obtained from the object with fewest tiles reached as the simulations ended. In either case, then, a single object is output by the simulations.

Before discussing what the results of interest are for the individual at hand, it is important to recall from the definition of MTSP that $S$, the seed, is part of what has to be determined. It would then seem like some strange dependency exists as far as the simulation is concerned, since it starts precisely with placing $S$ on the lattice while, to comply with the definition of MTSP, $S$ should be part of the simulation's output. What we do to avoid this is to assume, initially, that $S$ is some sort of universal seed, that is, a tile with wildcard labels on some of its sides (and $\epsilon$ on the others), each of which "becomes" the appropriate label of whatever other tile is accreted onto $S$ through it. At the end of the simulation, $S$ is the tile obtained from the original, universal $S$ by substituting the label $\epsilon$ for all wildcard labels that remain.

Let $i$ denote the individual under consideration and $\omega(i)$ the object output by the simulations on $i$. The simulations' results can be summarized as follows:

- The number of tile types actually used in assembling $\omega(i)$, denoted by $\theta(i)$. We have $\theta(i) \geq 0$, with $\theta(i)=0$ corresponding to the case in which $\omega(i)$ comprises $S$ only.

- The number of tiles that constitute $\omega(i)$, denoted by $|\omega(i)|$. We have $\omega(i) \geq 1$.

- The maximum number of tiles that $\omega(i)$ and $\omega^{*}$ (the object having the desired shape) may have in common under all possible superpositions of the two, including object rotations. The resulting number is denoted by $\kappa(i)$. We have $\kappa(i) \geq 1$, with $\kappa(i)=1$ corresponding to the case in which $\omega(i)$ comprises $S$ only or $\omega^{*}$ is one single tile. 
- The number of alternative tile types that could have been used at each step of the construction of $\omega(i)$ from the $S$ that resulted at the end of the simulation, all else unchanged up to that step, provided $\theta(i)>0$. In order to be an alternative tile type at step $u$, a tile type must be one of the $\theta(i)$ that were actually used along the simulation, must be different than the one that was actually used at step $u$, and finally must not be equivalent to the tile type that was used at step $u$ under rotation (if rotation is allowed by the model in use). This number is denoted by $\alpha(i)$. We have $\alpha(i) \geq 0$, with $\alpha(i)=0$ indicating the existence of one single accretive sequence leading from $S$ to $\omega(i)$ and using solely tiles of the $\theta(i)$ types used in the simulation. However, $\alpha(i)>0$ does not indicate that the other possible sequences lead to objects that have shapes other than that of $\omega(i)$. If $\omega(i)$ is a terminal object and has the same shape as $\omega^{*}$, then we are left with a sufficient condition-i.e., $\alpha(i)=0$ - for the termination and unicity that constraints $\mathrm{C} 1$ and $\mathrm{C} 2$ require, respectively, to hold for the tile-type set comprising the type of $S$ and the other $\theta(i)$ used in the simulation.

These are the results that get combined into fitness components for the evaluation of $i$. In addition to them, the following further result is used in ensuring well-formed offspring after crossover (henceforth, the set of tile types for which an individual stands is viewed as arranged in a sequence):

- The active region of $i$, given by the sub-sequence delimited on the left by the leftmost tile type actually used in assembling $\omega(i)$ and on the right by the rightmost tile type actually used. There may be unused tile types between the two delimiters.

\subsection{Fitness Components and Layering}

We use three fitness components in the evaluation of individual $i$, all three real functions mapping into the interval $[0,1]$ in such a way that closer to 1 is better. The first one is denoted by $f(i)$ and seeks to reflect the objective of MTSP, which is to require as small a tile-type set as possible. This fitness component is given by

$$
f(i)=1-\frac{1+\theta(i)}{|\omega(i)|}
$$

where the extra 1 in the fraction's numerator is meant to stand for the tile type of the seed $S$. Clearly, the worst that can happen to $f(i)$ is for each of the $|\omega(i)|$ tiles to be of a distinct type, in which case $1+\theta(i)=|\omega(i)|$ and $f(i)=0$. Otherwise, we always have $1+\theta(i)<|\omega(i)|$ and then $0<f(i)<1$.

The remaining two fitness components, denoted by $g(i)$ and $h(i)$, are related to constraints $\mathrm{C} 1$ and $\mathrm{C} 2$, and therefore to ensuring that the process of selfassembly always terminates and does so at an object of the same shape as $\omega^{*}$. The fitness component $g(i)$ is given by

$$
g(i)=\frac{2 \kappa(i)}{|\omega(i)|+\left|\omega^{*}\right|}
$$


where $\left|\omega^{*}\right|$ is the number of tiles that constitute $\omega^{*}$. The first thing to notice is that, if $\omega(i)$ and $\omega^{*}$ have the same shape (including coinciding hollows, if any), then $g(i)=1$, since in this case $\kappa(i)=|\omega(i)|=\left|\omega^{*}\right|$. All other alternatives yield $0<g(i)<1$, as in such cases we have $\kappa(i)<|\omega(i)|$ or $\kappa(i)<\left|\omega^{*}\right|$. Additionally, if the simulations ended upon reaching objects of the maximum allowed number of tiles, then $\kappa(i) \leq\left|\omega^{*}\right|<|\omega(i)|$ (assuming the simulations are allowed to create objects with strictly more tiles than $\omega^{*}$, which is certainly the case in all our experiments). In this case $g(i)<1$, thus keeping the $g(i)=1$ goal out of reach whenever $\omega(i)$ is not a terminal object. It then follows that $g(i)=1$ implies that $\omega(i)$ is a terminal object having the same shape as $\omega^{*}$.

The third fitness component, $h(i)$, depends on the maximum number of equivalent tiles that exist, under rotation, for the model in use. We let this be given by the number $\rho$, which is equal to 1 in the $2 \mathrm{D}$ model, to 4 in the $2 \mathrm{DR}$ model, and to 24 in the 3DR model. This fitness component is given by

$$
h(i)= \begin{cases}0, & \text { if } \theta(i)=0 \\ 1-\frac{\alpha(i)}{\rho|\omega(i)|(1+\theta(i))}, & \text { if } \theta(i)>0 .\end{cases}
$$

Notice first that, if $\theta(i)=0$, then no tile has been accreted onto $S$ and we ascribe to $h(i)$ the lowest possible value. If $\theta(i)>0$, on the other hand, then $\alpha(i)$ is defined and we use its value in obtaining $h(i)$. Clearly, in this case $h(i)=1$ if and only if $\alpha(i)=0$, which in turn is a sufficient (although not necessary) condition for the set comprising the tile type of $S$ and the additional $\theta(i)$ tile types to satisfy constraints $\mathrm{C} 1$ and $\mathrm{C} 2$, provided $\omega(i)$ is terminal and has the same shape as $\omega^{*}$. Furthermore, it also holds that $0<h(i)<1$ if $\alpha(i)>0$, since $\alpha(i)<\rho|\omega(i)|(1+\theta(i))$. Note finally that, as in the case of $f(i)$, the 1 added to $\theta(i)$ is meant to account for $S 5$

Our three fitness components are then seen to provide some measure of coverage of MTSP's objective and also of its constraints C1 and C2: $f(i)$ is concerned with minimizing supply diversity while $g(i)$ and $h(i)$, when $g(i)=$ $h(i)=1$, ensure that constraints $\mathrm{C} 1$ and $\mathrm{C} 2$ are satisfied by the set of $1+\theta(i)$ tile types revealed by the simulations of individual $i$. It would seem, then, that constraint $\mathrm{C} 3$, the one that requires full objects to be constructed, is so far unattended. While it is true that none of the fitness components refers to this constraint, our reason for proceeding in this way has been both pragmatic and a consequence of our design up to now. It has been pragmatic because we have found a further fitness component to account for C3 to be unnecessary in our experiments. Furthermore, and more importantly, constraint C3 is already taken into account, albeit indirectly, in our procedure for the simulation of an individual. This can be seen by recalling that attempts are made at filling hollows during simulation, and that selecting the matching pair for addition to the object at each step of the simulation privileges those pairs that will establish

\footnotetext{
${ }^{5}$ However, it is not in this case necessary, since the definition of $\alpha(i)$ does not consider substitutions for $S$. Keeping the 1 is harmless, however, and moreover lets both $f(i)$ and $h(i)$ be expressed as functions of the total number of tile types.
} 
the greater number of bonds, which is in accordance with what is required by constraint C3.

It will also become apparent from the computational experiments we describe that $f(i), g(i)$, and $h(i)$ do not always vary in consonance with one another. This has been the central motivation for us to pursue a multi-objective approach rather than try and combine the three fitness components into one single fitness function. What is left to specify, then, is how we use them in organizing individuals into layers of dominance. As we mentioned earlier, we use a Pareto-like criterion: it differs from traditional Pareto dominance in the sense that it distinguishes $f(i)$ from both $g(i)$ and $h(i)$, since $f(i)$ is the only one of the three that is unrelated to one of the problem's constraints, therefore unrelated to the feasibility of individual $i$.

Our layering criteria are then the following. If $i_{1}$ and $i_{2}$ are distinct individuals of the same population, then $i_{1}$ is said to dominate $i_{2}$ if $i_{1}$ is strictly better than $i_{2}$ according to $g$ and no worse according to $h$, or if $i_{1}$ is strictly better than $i_{2}$ according to $h$ and no worse according to $g$, or yet if they are indistinguishable from each other by $g$ or $h$ but $i_{1}$ is strictly better than $i_{2}$ according to $f$. More formally, $i_{1}$ dominates $i_{2}$ if and only if one of the following three conditions holds:

$$
\begin{gathered}
g\left(i_{1}\right)>g\left(i_{2}\right) \text { and } h\left(i_{1}\right) \geq h\left(i_{2}\right) ; \\
g\left(i_{1}\right) \geq g\left(i_{2}\right) \text { and } h\left(i_{1}\right)>h\left(i_{2}\right) ; \\
g\left(i_{1}\right)=g\left(i_{2}\right) \text { and } h\left(i_{1}\right)=h\left(i_{2}\right) \text { and } f\left(i_{1}\right)>f\left(i_{2}\right) .
\end{gathered}
$$

We then place strictly more weight on feasibility than on optimality.

\subsection{Crossover and Mutation}

The method we use for doing the crossover of two individuals $i_{1}$ and $i_{2}$ aims both at handling the size variability of the individuals and at ensuring that each of the two offspring inherits material from inside the active regions of both $i_{1}$ and $i_{2}$. The way this is achieved is by combining two well-known techniques. First the two individuals are aligned to each other at some randomly chosen position in such a way that the smallest one is contained inside the other. Then two crossover points are selected randomly, provided one intersects the active region of $i_{1}$ and the other that of $i_{2}$. Two-point crossover is then performed as illustrated in Figure 5. It is simple to see that the two resulting offspring have sizes that necessarily fall between the allowed minimum and maximum.

Whenever it has been decided that individuals will be selected to undergo crossover, we allow a fixed number of trials to be attempted so that the two individuals are not closer, by Euclidean distance in the three-dimensional space of the fitness components, than a given distance. This gives us a handle on trying to make sure that crossover is performed on sufficiently different individuals even when they both come from the same layer. In the event that no pair is found with the desired characteristic, crossover is performed on the last pair to have been selected. 
(a)

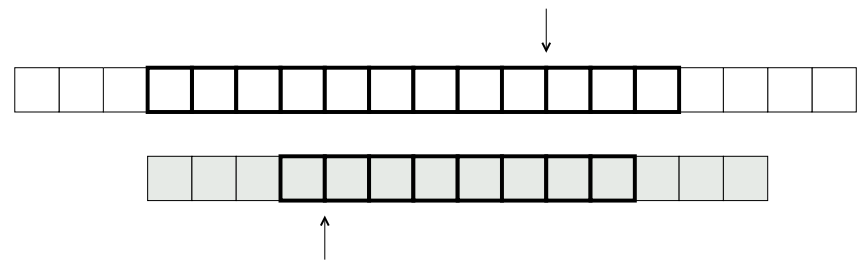

(b)
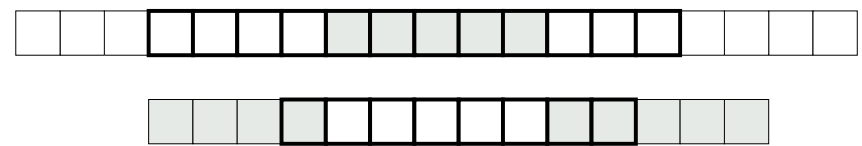

(c)
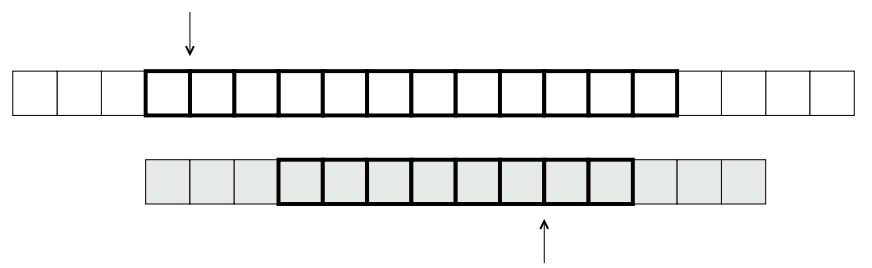

(d)

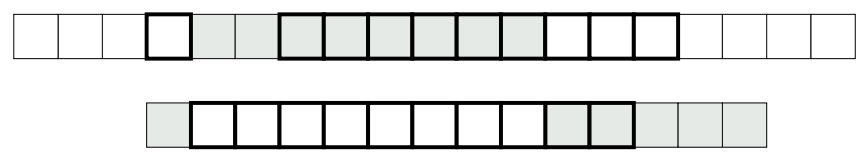

Figure 5: The crossover operator, applied to the individuals in (a) to produce those in (b), and to the ones in (c) to produce those in (d). Arrows indicate the crossover points. Thick-line borders are used to indicate the tile types in a parent's active region. 
Table 1: Common parameter values

\begin{tabular}{lc}
\hline Parameter & Value \\
\hline Number of generations $(G)$ & 1000 \\
Population size & 1000 \\
Elitist fraction & 0.1 \\
Diversity-preserving fraction & 0.05 \\
Initial crossover probability $\left(p_{1}\right)$ & 0.3 \\
Final crossover probability $\left(p_{G}\right)$ & 0.7 \\
Maximum number of simulations per individual & 10 \\
Max. number of crossover attempts per decision to do crossover & 1000 \\
\hline
\end{tabular}

Mutation is straightforward. One of the individual's tile types is selected at random, then one of its sides also at random. The chosen side's label is then replaced by another one from the $\tau$-dependent label table. The replacing label is also chosen at random, the only requirement being that it differ from the one it is replacing. If in the $2 \mathrm{DR}$ or $3 \mathrm{DR}$ model, then as mentioned earlier a polarity is randomly chosen for the label.

\section{Computational Results}

We have conducted extensive experimentation with the evolutionary heuristic of Section 3, allowing for several combinations of its parameters. Here we report on some of the most successful and, occasionally, also on some of the experiments that, though not completely successful, have shed some light on parameter choice in a significant way. In all experiments in two dimensions $\omega^{*}$ has been an $n \times n$ square with different choices for the value of $n$. In three dimensions, we have concentrated on the $5 \times 5 \times 5$ cube. Most parameter values have been the same in all experiments and appear in Table 1.

Most experiments have also used temperature $\tau=2$, a notable exception being one of the three-dimensional experiments. The seed $S$ used in all twodimensional experiments has two wildcard labels placed so that $S$ is one of the corners of the square. In three dimensions $S$ has three wildcard labels, again intended to allow the seed to occupy one of the cube's corners.

Our results for the 2D model refer to $n=5$ or $n=15$ (but see also Section 5 , where we discuss the $n=25$ case as well) with the following additional parameter values. For $n=5$, a $30 \times 30$ lattice, simulated objects of no more than 100 tiles, the size of an individual between 25 and 50, and a label table with 10 non- $\epsilon$ labels. For $n=15$, these become $45 \times 45,900,50-100$, and 20, respectively.

For $n=5$ we had several successful runs, a success being characterized by the occurrence of at least one individual $i$ for which $g(i)=h(i)=1$ (so the $1+\theta(i)$ tile types satisfy constraints $\mathrm{C} 1$ and $\mathrm{C} 2$ ), and for which constraint $\mathrm{C} 3$ is also satisfied, and furthermore $1+\theta(i)$, the number of tile types (including that of $S$ ), is as indicated in (11). These runs correspond to the various combinations of 0 or 
0.01 as the minimum distance between individuals for crossover to be performed with $W_{1}=W_{G}=15$ or $W_{1}=1, W_{G}=30$ (recall the latter regulate the selection of layers through (3)). For the particular choice of 0 as minimum distance, success was achieved in all runs of a series of five with each of $W_{1}=W_{G}=15$ or $W_{1}=1, W_{G}=30$. On average, this happened after about 502 and 433 generations, respectively. One unexpected (and, in fact, unsought for) outcome related to one of the $W_{1}=W_{G}=15$ runs is that the resulting number of distinct labels is $n+2=7$, better therefore than the best known estimate, which is $n+3$ [16] 6

The solution achieved by this latter run is shown in Figure 6. The corresponding evolution plots for the three fitness components are shown in Figure 7. These plots were obtained according to the following (somewhat arbitrary) method, which holds also for all other similar plots in the sequel. For each generation, the fitness component $g$ is plotted for the best individual found so far in terms of its $g$ value ( $h$ and $f$ are used, in this order, for tie breaking). For this best individual, $h$ and $f$ are then plotted. Notice, then, that the $g$ plot is necessarily nondecreasing, while the plots for $h$ and $f$ may oscillate.

The $n=15$ case also yielded plenty of successes under the same parameter choices described above for $n=5$ with regard to the minimum distance for crossover and the values of $W_{1}$ and $W_{G}$. Now, however, choosing the distance to be 0.01 yielded success in all runs in a series of five for $W_{1}=W_{G}=15$ and another five for $W_{1}=1, W_{G}=30$. The solution achieved by one of the runs with $W_{1}=1, W_{G}=30$ is shown in Figure 8, with fitness-component plots in Figure 9. When compared to the plots of Figure 7 the ones in Figure 9 reflect the increased difficulty that comes from moving from $n=5$ to $n=15$, since the solution is approached much more slowly for the larger square.

As we remarked earlier, as we move from the $2 \mathrm{D}$ model to $2 \mathrm{DR}$ we expect to be afforded greater flexibility and therefore greater ease in obtaining success with our evolutionary heuristic for MTSP. Here we illustrate this for the $n=5$ case, using the same parameters as for the case singled out above at the end of our discussion of the 2D model. Success was obtained in all of five runs. One of them is illustrated in Figures 10 and 11, containing respectively the solution that was achieved and the fitness-component plots. From the latter, in particular, the expected increased ease in finding the solution emerges very clearly, since convergence to 1 of both $g$ and $h$ occurs relatively early in the evolution.

It is curious to observe, in Figure 10, that the number of tile types is $n+1=6$, therefore significantly smaller than the known upper bound given in (1) for the $2 \mathrm{D}$ model, which for $n=5$ is $n+4=9$. Of course, such an upper bound may turn out to be strictly looser for the 2DR model than it is for the 2D model once similar properties become known for the 2DR model. Until then, we are left with this one source of assessment of the solution shown in the figure, that is, by comparison with the $2 \mathrm{D}$ model.

\footnotetext{
${ }^{6}$ Minimizing the number of distinct labels is not part of MTSP and we are therefore generally unconcerned with it in this paper. However, this is an additional goal for which plausible arguments exist [7, so our find, although serendipitous, amounts to an interesting by-product.
} 
As we switch to three dimensions and adopt the 3DR model, solutions are still obtained for parameter combinations similar to the ones considered so far under two dimensions, but they no longer constitute successes in the sense we have established. The reason for this is that, even though a full $5 \times 5 \times 5$ cube is obtained, its fullness depends on the order of assembly and therefore constraint C3 is not satisfied. Our suspicions as to why this occurs fell, naturally, on the value of $\tau$, so far kept constant at 2 . In fact, adopting $\tau=3$ has yielded the desired solution, obtained in one single run and shown in Figure 12. Once again, it is curious to note the $n+2=7$ tile types used and to compare this figure to both the prediction of (11) and the results discussed heretofore. Parameter values were the following: a $30 \times 30 \times 30$ lattice, simulated objects of no more than 900 tiles, individuals sized between 25 and 35, 20 non- $\epsilon$ labels available for selection, minimum distance for crossover of 0.01 , and finally $W_{1}=1, W_{G}=30$. The corresponding plots of the evolution of the fitness components are given in Figure 13 
(a)
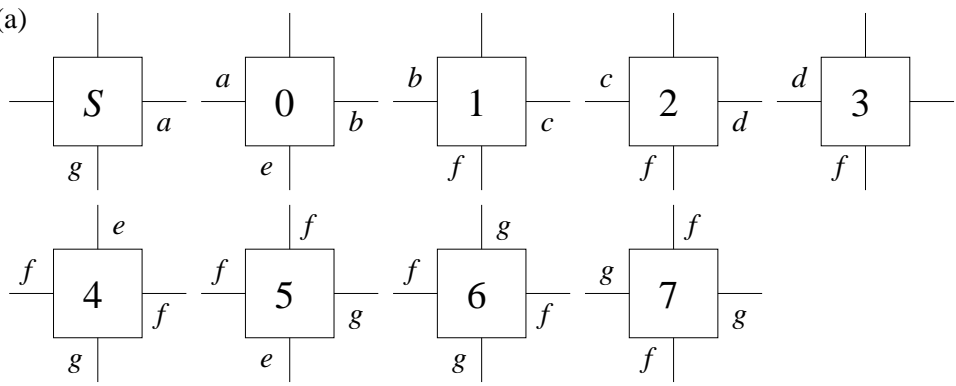

(b)

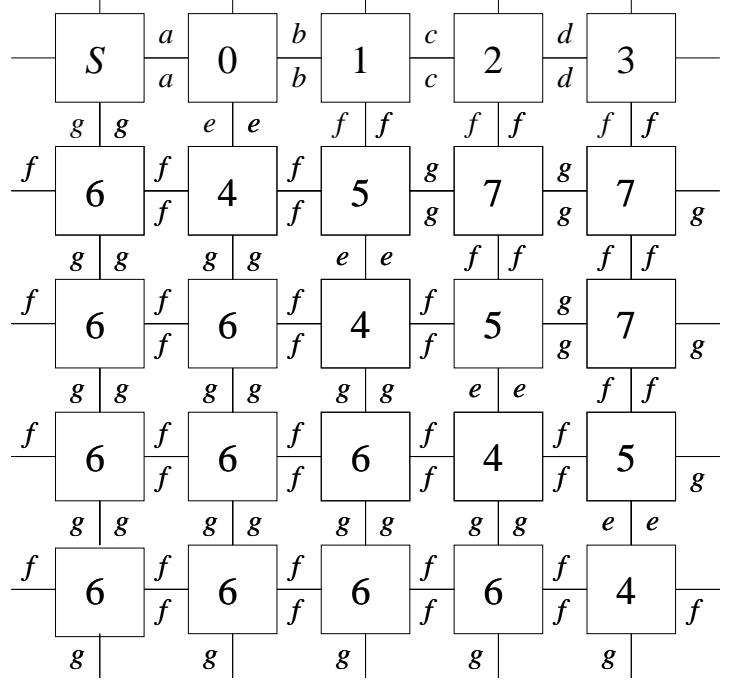

Figure 6: A solution obtained in the 2D model for $n=5$. Tile types appear in (a), the final object in (b). Temperature is $\tau=2$. Label intensities are $I(a)=\cdots=I(e)=2$ and $I(f)=I(g)=1$. 


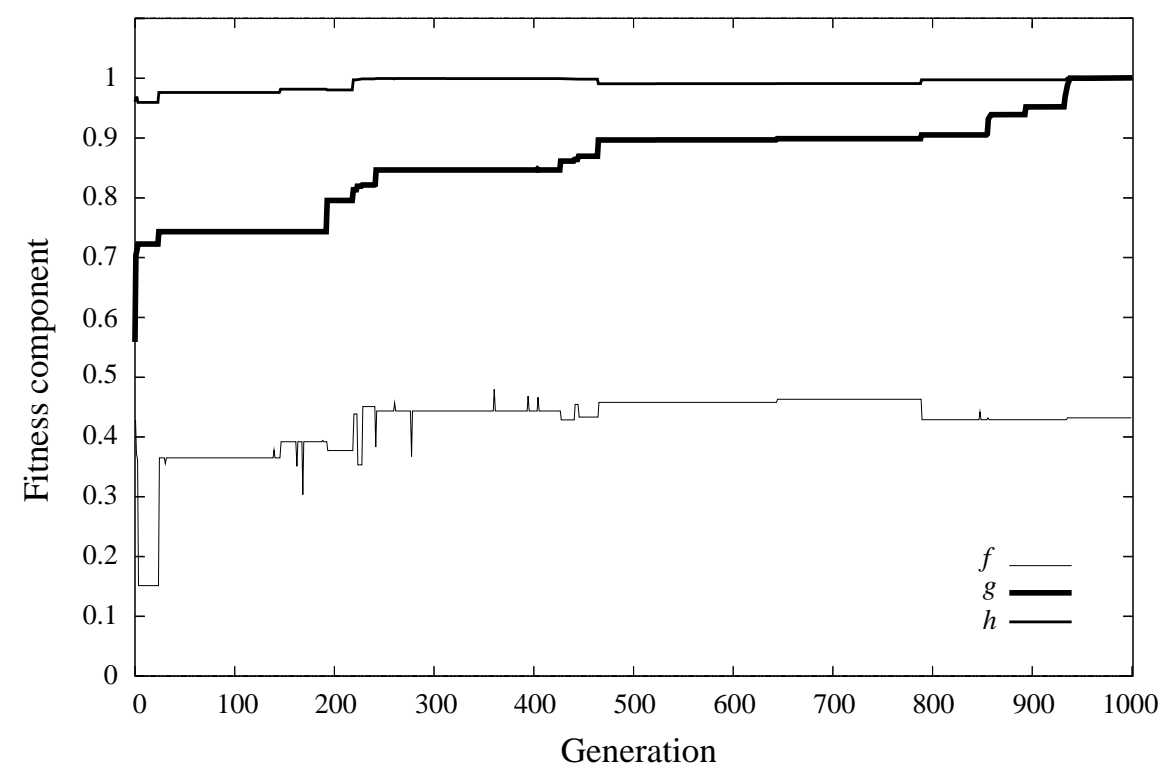

Figure 7: Evolution of the fitness components for the solution shown in Figure 6. 
(a)

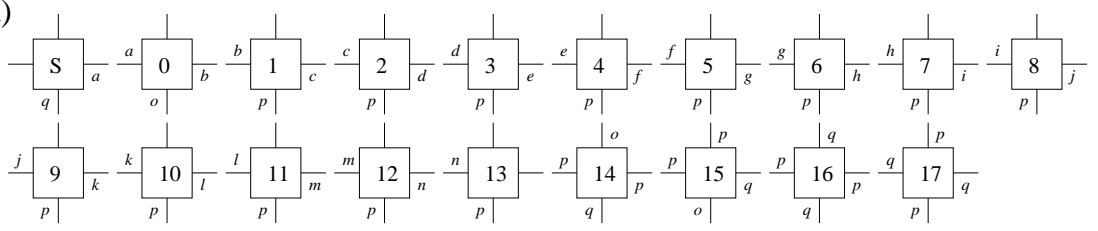

(b)

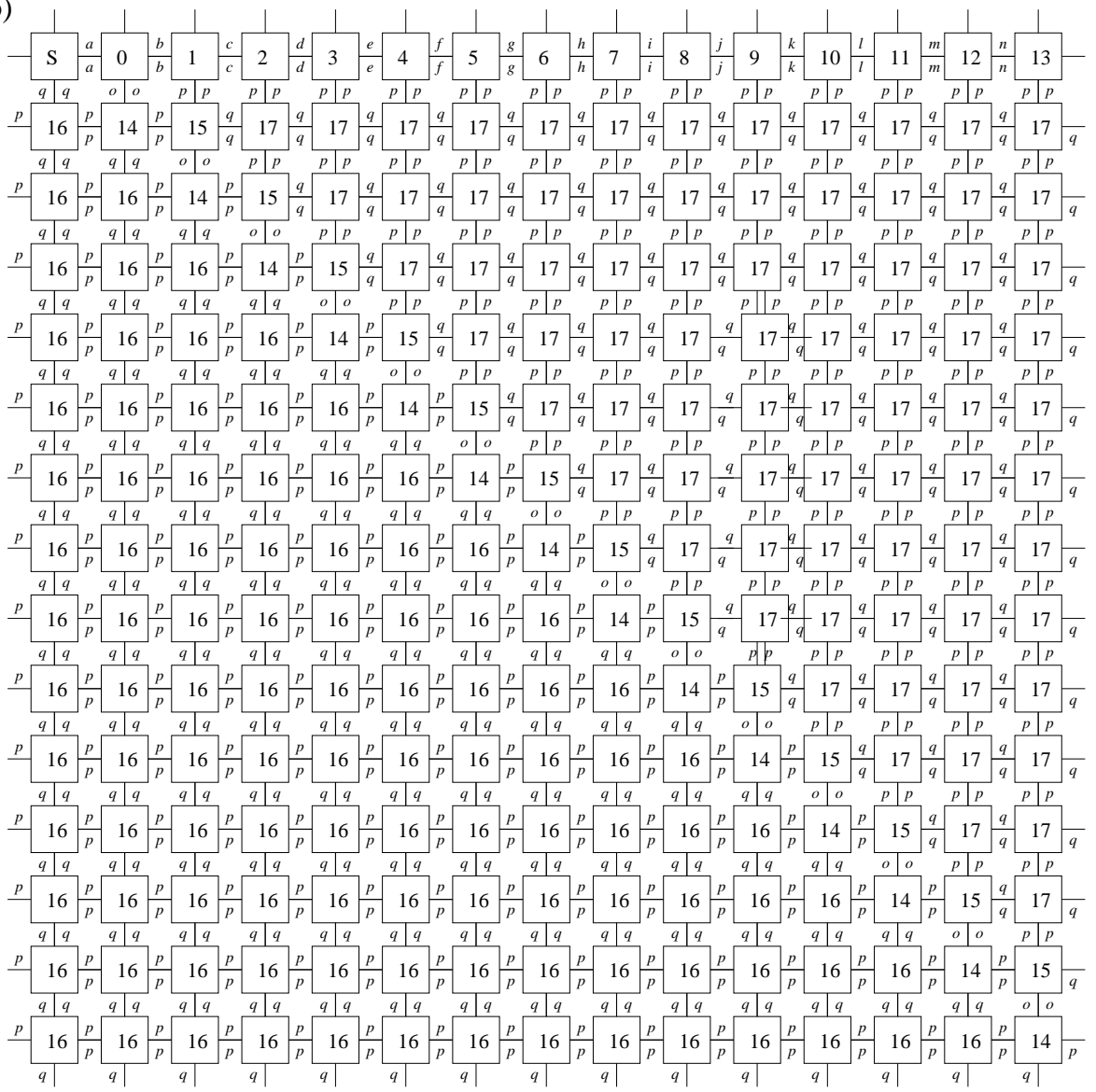

Figure 8: A solution obtained in the 2D model for $n=15$. Tile types appear in (a), the final object in (b). Temperature is $\tau=2$. Label intensities are $I(a)=\cdots=I(o)=2$ and $I(p)=I(q)=1$. 


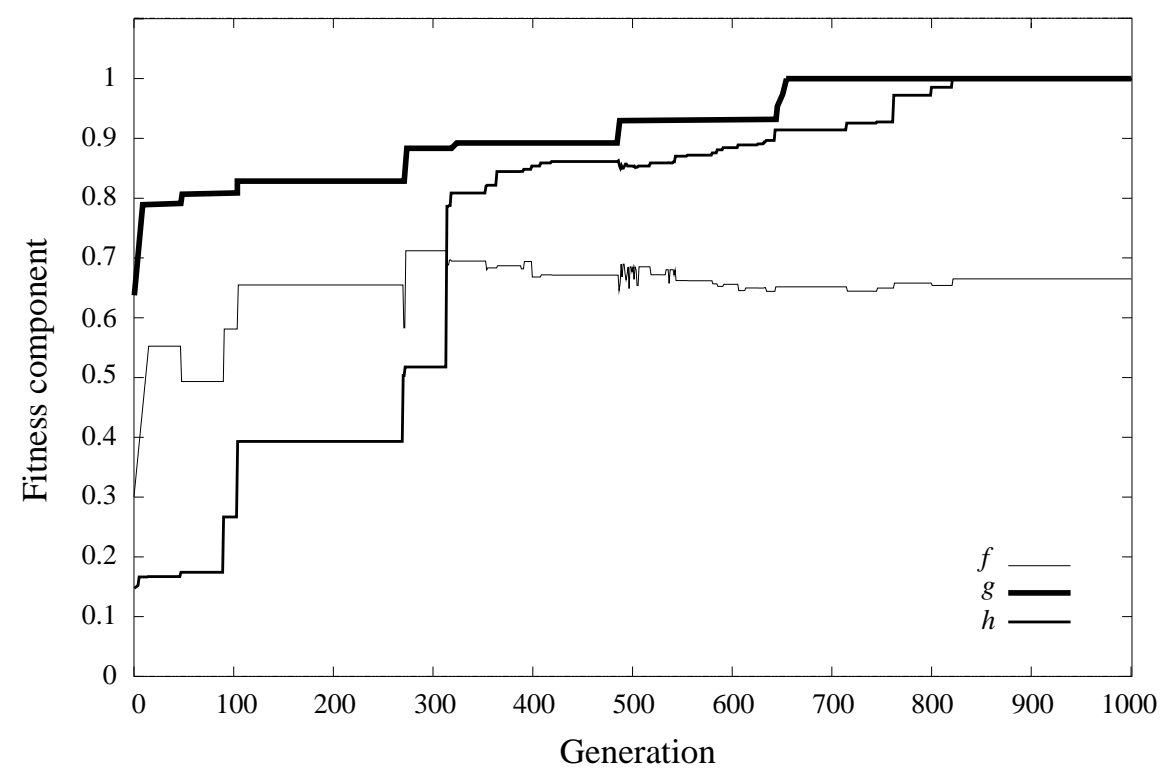

Figure 9: Evolution of the fitness components for the solution shown in Figure 8 

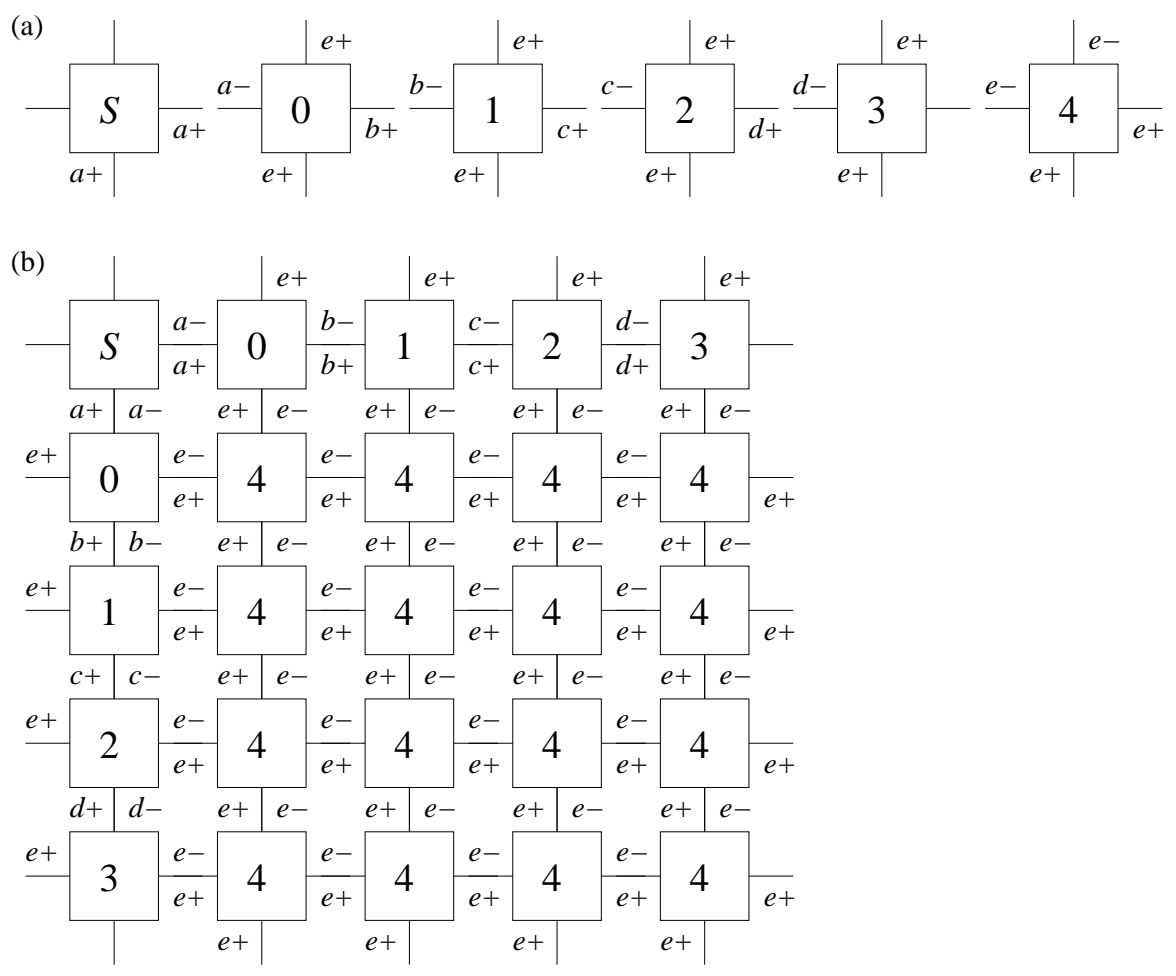

Figure 10: A solution obtained in the 2DR model for $n=5$. Tile types appear in (a), the final object in (b). Temperature is $\tau=2$. Label intensities are $I(a)=\cdots=I(d)=2$ and $I(e)=1$. 


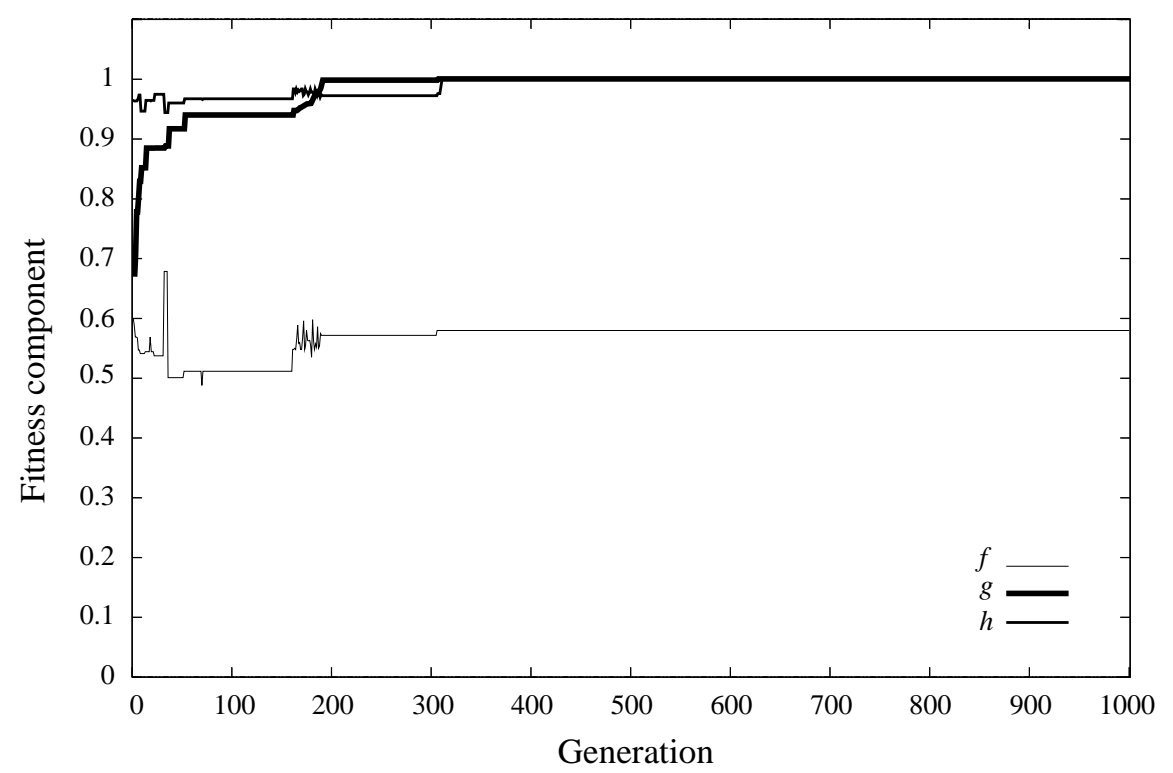

Figure 11: Evolution of the fitness components for the solution shown in Figure 10 . 
(a)

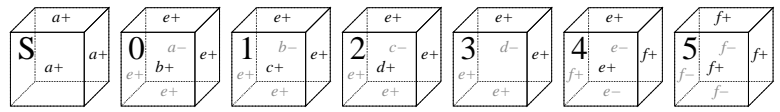

(b)
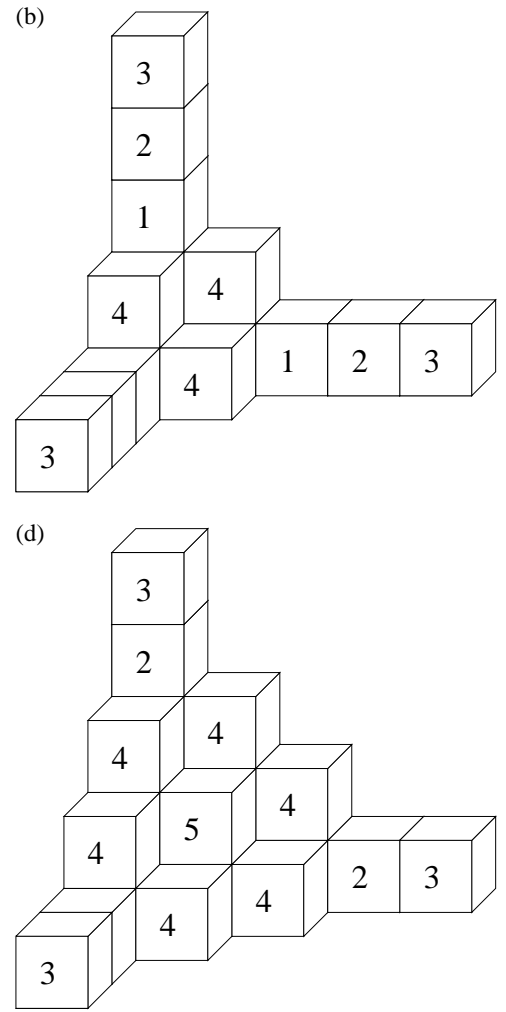

(c)

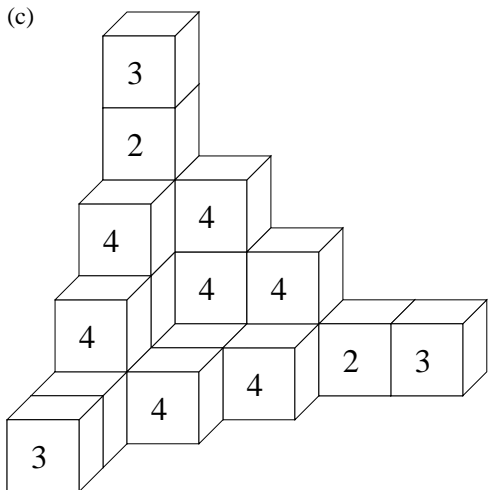

Figure 12: A solution obtained in the 3DR model for $n=5$. Tile types appear in (a), three intermediate objects on the sequence leading to the final $5 \times 5 \times 5$ cube appear in (b)-(d). Temperature is $\tau=3$. Label intensities are $I(a)=$ $\cdots=I(d)=3, I(e)=2$, and $I(f)=1$. In (b), the seed $S$ occupies the hidden corner of the cube and three tiles of type 0 (also hidden) are accreted onto it. Then follow tiles of types 1, 2, and 3 along each dimension. The cube is completed by filling with tiles of type 4 the three faces on which such tiles are already present, and finally placing type- 5 tiles in all remaining positions. 


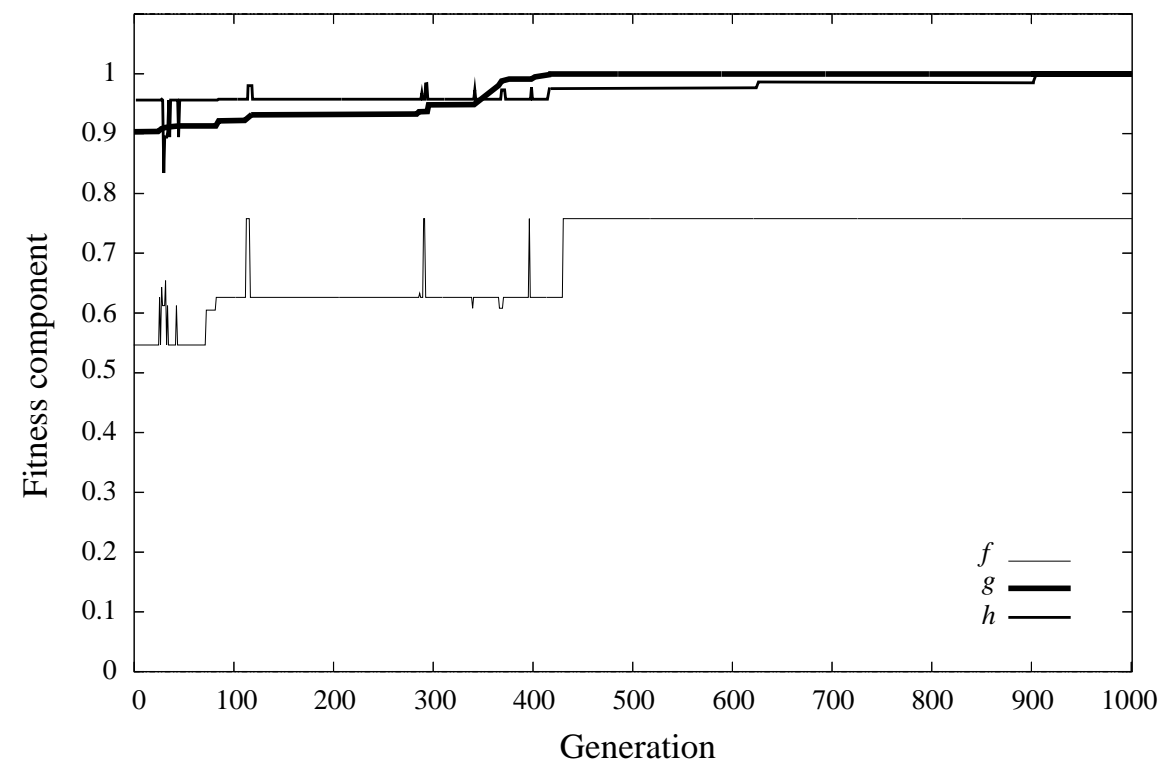

Figure 13: Evolution of the fitness components for the solution shown in Figure 12 
Table 2: Average running times

\begin{tabular}{lrr}
\hline Experiment & Number of cores & Time (hours) \\
\hline 2D model, $n=5$ & 8 & 4.0 \\
2D model, $n=15$ & 10 & 7.7 \\
2DR model, $n=5$ & 12 & 1.5 \\
3DR model, $n=5$ & 12 & 12.0 \\
\hline
\end{tabular}

\section{Discussion and Concluding Remarks}

One practical measure of the computational hardness of solving MTSP is the time it has taken our heuristic to complete for each of the runs described in Section 4. Like most evolutionary algorithms, our heuristic has a tremendous potential for parallel execution, not only because individuals may be evaluated independently of one another, but especially because it is precisely in computing the fitness components that lies most of the difficulty. We ran our experiments on a small local grid with exclusive access to all hosts, each running a Linux operating system on an Intel Pentium D (a two-core processor) at $2.8 \mathrm{GHz}$ with 2GB of RAM. Average running times are given in Table 2 .

In view of the times given in the table, it comes as no surprise that our search for appropriate parameter-value combinations has of necessity been severely limited. This is often the case with evolutionary approaches, and no doubt the availability of larger grids would instantly provide many fresh opportunities for tune-up and performance studies. However, we believe to have already provided a sort of proof of principle regarding the possibility of using an evolutionary approach to tackle MTSP and perhaps other hard problems related to selfassembly systems.

We find, then, that further effort will be more profitably spent if directed toward understanding other aspects of MTSP with the use of heuristics like the one we have introduced. We finalize by briefly reporting on one step we have already taken in such a direction, having to do with the study of the so-called complex seeds, i.e., seeds that, unlike the $S$ we have used throughout, comprise more than one tile [16. The initial motivation came from our heuristic's apparent inability to complete any successful runs in the 2D model for $n=25$ using various combinations of parameter values. Interestingly, though, once the possibility of a complex seed was brought into the scene it became possible for our heuristic to conclude successful runs with parameter values very similar to those used in Section 4

The result for one of the successful runs is shown in Figure 14, where the solution is given along with the complex seed that was used 7 and in Figure 15 . which contains the fitness-component plots. Even with the use of the complex seed, though, comparing these plots with those of Figure 9, relative to the

\footnotetext{
${ }^{7}$ This seed is based on the solution to MTSP for $n=4$, for which (1) predicts as an upper bound exactly the $n+4=8$ tile types that it contains. It has wildcard labels on all tile sides that face inward with respect to the final square.
} 
$n=15$ case in the $2 \mathrm{D}$ model, reveals the increased hardness due to the greater value of $n$, as convergence to 1 of the $g$ and $h$ fitness components occurs more slowly. For this run with $n=25$ we used the same basic set of parameters as in Section 4 for the 2D cases, with the following differences/enlargements: fixed crossover probability of $p_{1}=p_{G}=0.3$, a $100 \times 100$ lattice, simulated objects of no more than 2500 tiles, individuals sized between 100 and 150, 20 non- $\epsilon$ labels available for selection, minimum distance for crossover of 0.01 , and finally $W_{1}=1, W_{G}=30$. The average running time was of 10 hours on 10 cores. 
(a)

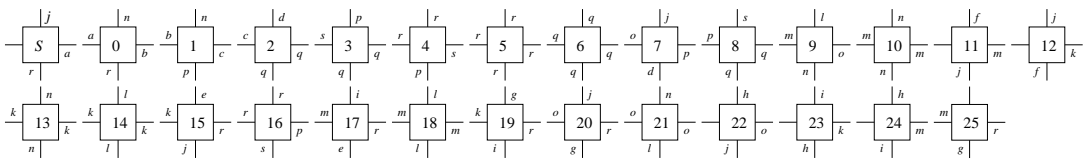

(b) $-\frac{1}{24}-\frac{1}{10}-\frac{1}{18}-\frac{1}{25}-\frac{1}{5}-\frac{1}{5}-\frac{1}{5}-\frac{1}{5}-\frac{1}{5}-\frac{1}{5}-\frac{1}{5}-\frac{1}{5}-\frac{1}{5}-\frac{1}{5}-\frac{1}{5}-\frac{1}{5}-\frac{1}{5}-\frac{1}{5}-\frac{1}{5}-\frac{1}{5}-\frac{1}{5}-\frac{1}{5}-\frac{1}{5}-\frac{1}{16}-\frac{1}{8}-$

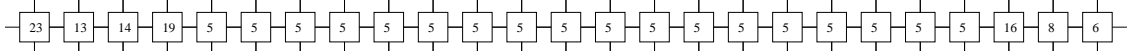
$-24-10-18-17-5-5-5-5-5-5-5-5-5-5-5-5-5-5-5-5-5-16-5-6-$

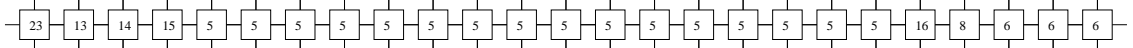

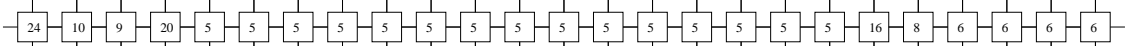

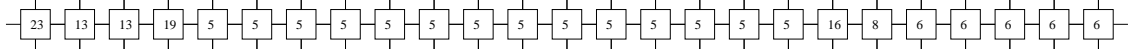
$-24-10-10-17-5-5-5-5-5-5-5-5-5-5-5-5-5-16-8-6-6-6-6-6-$

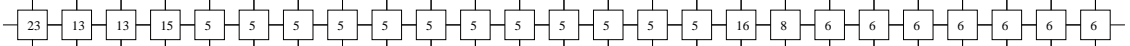

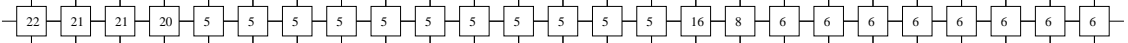

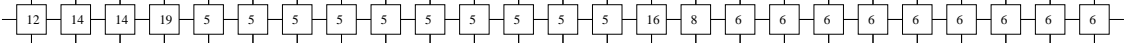

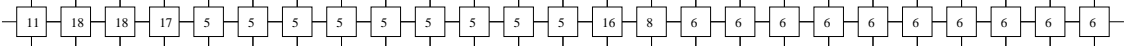
$-12-14-14-15-5-5-5-5-5-5-5-5-16-\sqrt{5}-6-6 \sqrt{6}-6-6$

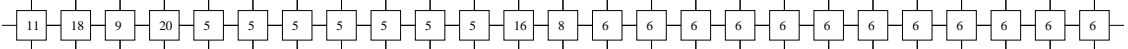

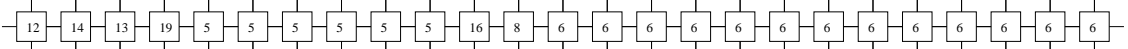
$-110-10-17-5-5-5-5-5-16-8-6-6-6-6 \sqrt{6}-6-6$

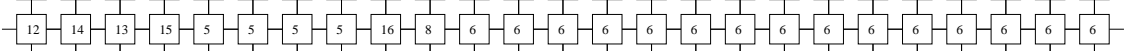

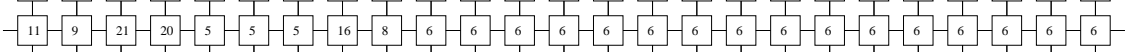

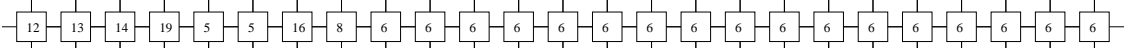

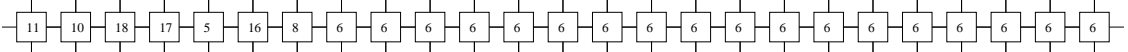
- $12-13-14-15-16-8-6-6-6-6-6-6-6)$

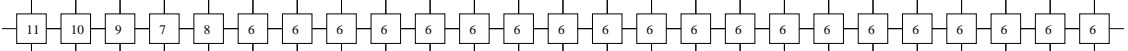

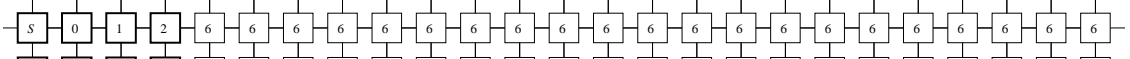

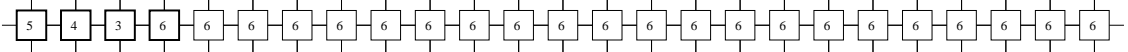

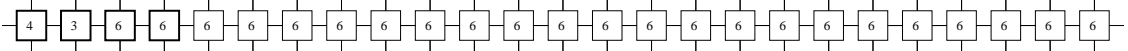

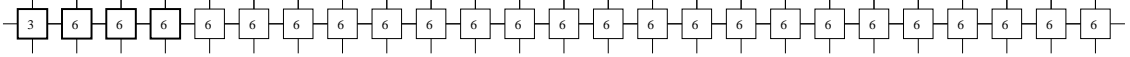

Figure 14: A solution obtained in the 2D model for $n=25$. Tile types appear in (a), the final object in (b). The complex seed used is shown in part (b) comprising all sixteen tiles with thick-line borders. Temperature is $\tau=2$. Label intensities are $I(a)=\cdots=I(h)=I(p)=2$ and $I(i)=\cdots=I(o)=$ $I(q)=I(r)=1$. 


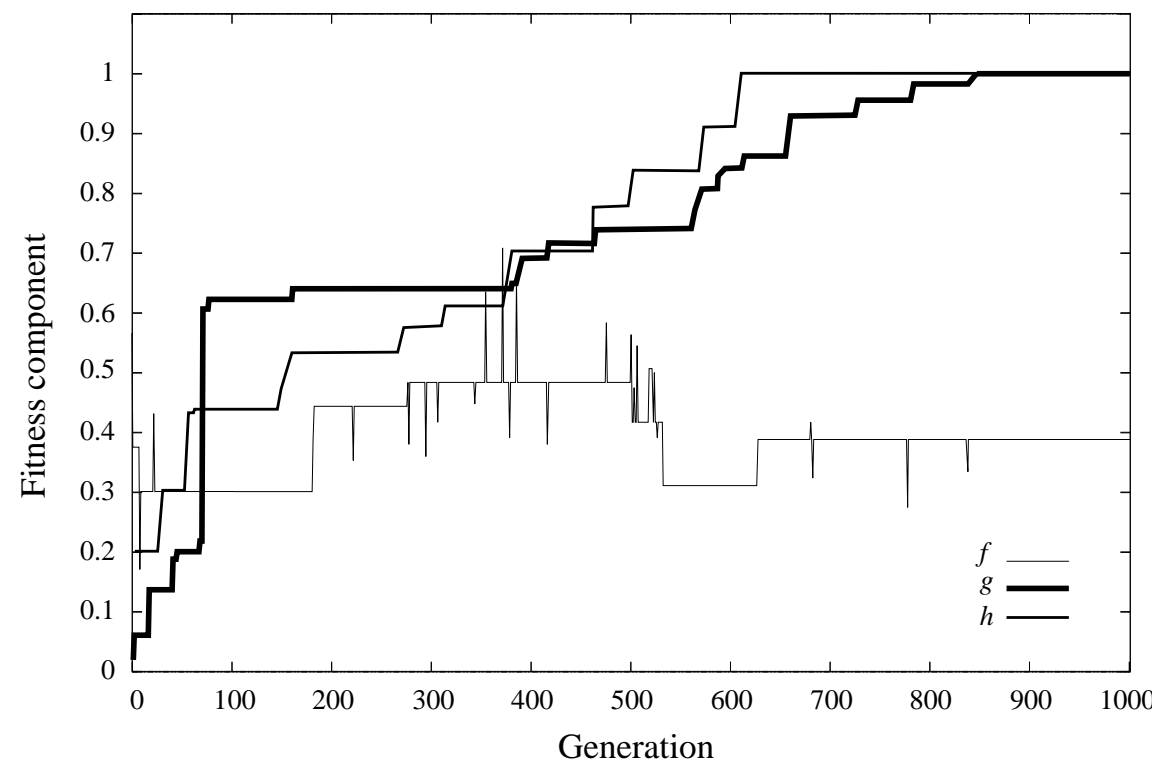

Figure 15: Evolution of the fitness components for the solution shown in Figure 14. 


\section{Acknowledgments}

The authors acknowledge partial support from CNPq, CAPES, and a FAPERJ BBP grant.

\section{References}

[1] L. Adleman, Q. Cheng, A. Goel, M.-D. Huang, D. Kempe, P. M. de Espanes, and P. W. K. Rothemund. Combinatorial optimization problems in self-assembly. In Proceedings of 34th Annual ACM Symposium on Theory of Computing, pages 23-32, 2002.

[2] G. Aggarwal, Q. Cheng, M. H. Goldwasser, M.-Y. Kao, P. M. de Espanes, and R. T. Schweller. Complexities for generalized models of self-assembly. SIAM Jornal on Computing, 34:1493-1515, 2005.

[3] S. Angelov, S. Khanna, and M. Visontai. On the complexity of graph selfassembly in accretive systems. In C. Mao and T. Yokomori, editors, DNA Computing, volume 4287 of Lecture Notes in Computer Science, pages 95110, Berlin, Germany, 2006. Springer.

[4] Y. Baryshnikov, E. Coffman, and P. Momcilovic. Self assembly times in DNA-based computation. SIGMETRICS Performance Evaluation Review, $32: 35-37,2004$.

[5] Y. Brun. Adding and multiplying in the tile assembly model. In Proceedings of the 4th Foundations of Nanoscience: Self-Assembled Architectures and Devices, 2007.

[6] H.-L. Chen, Q. Cheng, A. Goel, M.-D. Huang, and P. M. de Espanes. Invadable self-assembly: combining robustness with efficiency. In Proceedings of the 15th Annual ACM-SIAM Symposium on Discrete Algorithms, pages 890-899, 2004.

[7] G. M. Cooper and R. E. Hausman. The Cell: A Molecular Approach. ASM Press and Sinauer Associates, Washington, DC, 2 edition, 2003.

[8] B. Hogberg and H. Olin. Programmable self-assembly-unique structures and bond uniqueness. Journal of Computational and Theoretical Nanoscience, 3:391-397, 2006.

[9] D. D. Majumder, C. Ulrichs, D. Majumder, I. Mewis, A. R. Thakur, R. L. Brahmachary, R. Banerjee, A. Rahman, N. Debnath, D. Seth, S. Das, I. Roy, A. Ghosh, P. Sagar, C. Schulz, N. Q. Linh, and A. Goswami. Current status and future trends of nanoscale technology and its impact on modern computing, biology, medicine and agricultural biotechnology. In Proceedings of the International Conference on Computing: Theory and Applications, pages 563-573, 2007. 
[10] C. Pistol and C. Dwyer. Scalable, low-cost, hierarchical assembly of programmable DNA nanostructures. Nanotechnology, 18:125305, 2007.

[11] C. Pistol, A. R. Lebeck, and C. Dwyer. Design automation for DNA selfassembled nanostructures. In Proceedings of the 43rd Annual Conference on Design Automation, pages 919-924, 2006.

[12] J. H. Reif. The emerging discipline of biomolecular computation in the US. New Generation Computing, 20:217-236, 2002.

[13] J. H. Reif, S. Sahu, and P. Yin. Complexity of graph self-assembly in accretive systems and self-destructible systems. In A. Carbone and N. A. Pierce, editors, DNA Computing, volume 3892 of Lecture Notes in Computer Science, pages 257-274, Berlin, Germany, 2006. Springer.

[14] A. Requicha and D. Arbuckle. CAD/CAM for nanoscale self-assembly. IEEE Computer Graphics and Applications, 26:88-91, 2006.

[15] P. W. K. Rothemund. Folding DNA to create nanoscale shapes and patterns. Nature, 440:297-302, 2006.

[16] P. W. K. Rothemund and E. Winfree. The program-size complexity of selfassembled squares (extended abstract). In Proceedings of the 32nd Annual ACM Symposium on Theory of Computing, pages 459-468, 2000.

[17] C. E. Schafmeister. Molecular lego. Scientific American, 296:64-71, 2007.

[18] Y. Terada and S. Murata. Automatic assembly system for a largescale modular structure - hardware design of module and assembler robot. In Proceedings of the IEEE/RSJ International Conference on Intelligent Robots and Systems, volume 3, pages 2349-2355, 2004.

[19] G. Terrazas, N. Krasnogor, G. Kendall, and M. Gheorghe. Automated tile design for self-assembly conformations. In Proceedings of the IEEE Congress on Evolutionary Computation, volume 2, pages 1808-1814, 2005.

[20] E. Tuci, R. Gross, V. Trianni, F. Mondada, M. Bonani, and M. Dorigo. Cooperation through self-assembly in multi-robot systems. ACM Transactions on Autonomous and Adaptive Systems, 1:115-150, 2006.

[21] H. Wang. Proving theorems by pattern recognition. Bell Systems Technical Journal, 40:1-42, 1961.

[22] J. Werfel, Y. Bar-Yam, D. Rus, and R. Nagpal. Distributed construction by mobile robots with enhanced building blocks. In Proceedings of the IEEE International Conference on Robotics and Automation, pages 2787-2794, 2006. 
[23] E. Winfree. On the computational power of DNA annealing and ligation. In R. J. Lipton and E. B. Baum, editors, DNA Based Computers, volume 27 of DIMACS Series in Discrete Mathematics and Theoretical Computer Science, pages 199-221. American Mathematical Society, Providence, RI, 1996.

[24] E. Winfree. Algorithmic Self-Assembly of DNA. PhD thesis, California Institute of Technology, Pasadena, CA, 1998.

[25] E. Winfree. Self-healing tile sets. In J. Chen, N. Janoska, and G. Rozenberg, editors, Nanotechnology: Science and Computation, pages 55-78. Springer, Berlin, Germany, 2006.

[26] E. Winfree, F. Liu, L. A. Wenzler, and N. C. Seeman. Design and selfassembly of two-dimensional DNA crystals. Nature, 394:539-544, 1998.

[27] H. Yi, S. Nisar, S. Y. Lee, M. A. Powers, W. E. Bentley, G. F. Payne, R. Ghodssi, G. W. Rubloff, M. T. Harris, and J. N. Culver. Patterned assembly of genetically modified viral nanotemplates via nucleic acid hybridization. Nano Letters, 5:1931-1936, 2005. 